in order that the effect of jar should not enter into the experiment. They were then loaded with, respectively, in each set, $95,90,85,80$, $75,70,65,60,55$, per cent of the average ultimate strength, as already determined. This was done in November, 1881. Since that date, a number have broken, as follows : -

Effect of prolonged stress. - Swedish iron wire.

\begin{tabular}{|c|c|c|}
\hline \multirow{2}{*}{$\begin{array}{l}\text { Per cent max. } \\
\text { static load. }\end{array}$} & \multicolumn{2}{|c|}{ TIME UNDER STRESS. } \\
\hline & Hard wire (unannealed). & Soft (annealed). \\
\hline $\begin{array}{l}95 \\
90 \\
85 \\
80 \\
75 \\
70 \\
65 \\
60 \\
55\end{array}$ & $\begin{array}{l}80 \text { days. } \\
35 \text { days. } \\
17 \text { months, unbroken. } \\
91 \text { days. } \\
\text { Unbroken. } \\
\text { 6" } \\
\text { "6 } \\
\text { "6 }\end{array}$ & $\begin{array}{l}3 \text { minutes. } \\
5 \text { minutes. } \\
1 \text { day. } \\
266 \text { days. } \\
17 \text { “ } \\
455 \text { “" } \\
455 \text { " } \\
\text { Unbroken. }\end{array}$ \\
\hline
\end{tabular}

Thus, wire loaded with but 65 per cent of the breaking-load, as usually determined, broke after being subjected to stress for a period of fifteen months, when annealed; while hard wire carrying 85 per cent of the maximum temporary load remains unbroken after seventeen months. It is seen that these results are the same in kind as those obtained by Vicat, and confirm the conclusion that heavily loaded iron, as well as other metals and the woods, are likely to yield ultimately under loads that are sustained for short periods of time without apparent injury. This fact has been amply proven by earlier investigators, as well as by the writer; but the difference above observed, between hard and soft iron, has, so far as the writer has been able to learn, never, until now, been discovered.

Although the experiments of which this is the first are not yet concluded, this discovery, if such it prove, has seemed to be of sufficient importance to justify this note.

Hoboken, N.J., April 22, 1883.

R. H. Thurston.

ELEPHANTIASIS, OR FILARIA DISEASE.

Dr. A. F. A. KING, dean of the faculty of the National medical college, has recently cited a number of curious coincidences between the habits of the mosquito and the observed phenomena respecting malaria. There are, however, fatal objections to any theory that would connect the two ; the coincidences rather indicating that the germs of both develop in similar places. The connection of the mosquito as an intermediary host in the full life-development of the haematozoön, Filaria sanguinis-hominis, however, has been very fully and conclusively made out by Dr. Patrick Manson, of Amoy, China, in the Customs medical reports, published in Shanghai by the order of the inspector-general of customs. Dr. Manson discovered the parent Filaria in the mosquito in 1878 , and has since published several admirable articles, giving the results of his experiments ; which, in the main,

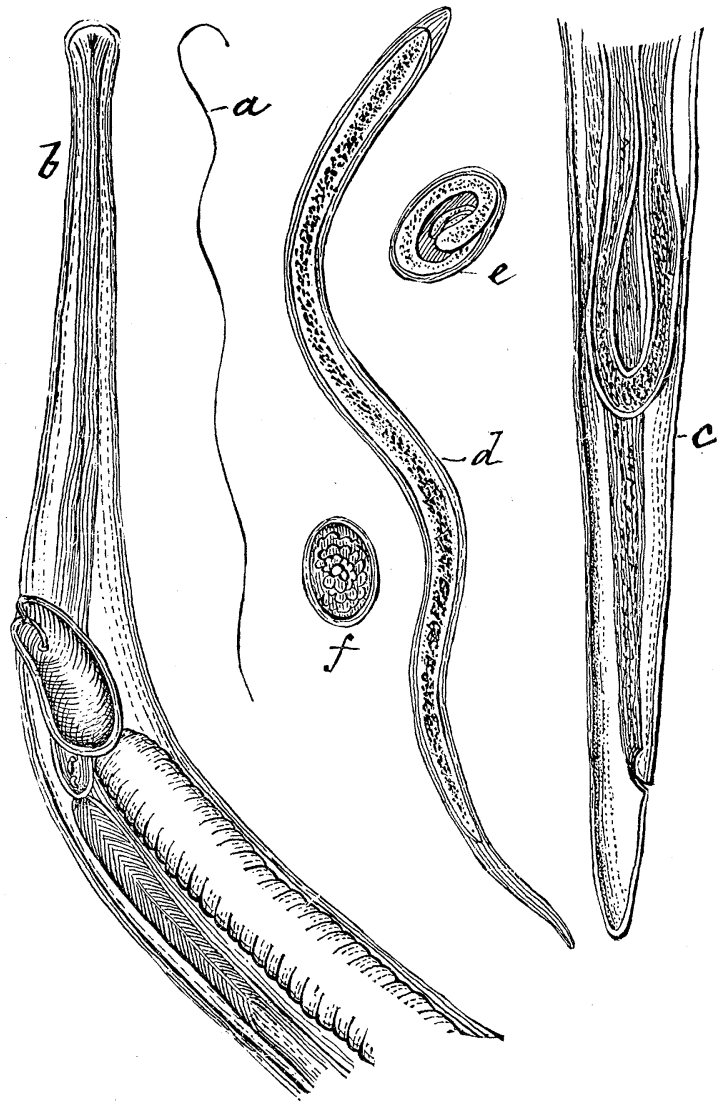

FILARIA BANCROFTI.

$a$, female (nat. size); $b$, head and neck ( $\times 55$ diam.) ; $c$, tail ; $d$, free embryo ( $\times 400$ diam.); $e$, egg containing an embryo; $f$ egg, with mulberry cleavage of the yolk ( $\times 360$ diam. $)-A f t e r$ egg, with

have been independently confirmed by Dr. Mackenzie of the London pathological society, Mr. T. R. Lewis in India, Dr. W. W. Myers, Drs. 'T. S. Cobbold, Wucherer, Bancroft, Araujo, and others. The facts have an entomological bearing, and are of great scientific interest and practical importance. They may be briefly stated as follows :-

In 1872 Dr. T. R. Lewis first announced the discovery of the immature or larval haematozoön, to which he gave the above trinomial 
term, in the blood and urine of persons afflicted with chyluria. The mature form was first described by Cobbold as Filaria Bancrofti, in 1877 (London lancet, Oct. 6, 1877). As found in the lymph, the parent Filaria emits her young in the lymph-stream. The young Filaria is an elongate, transparent, very active creature, measuring $\frac{1}{90} \times \frac{1}{3500}$. It makes its way from the lymph to the blood, where, however, it seems to undergo no growth or development. In this its new-born state it is enclosed in a delicate, transparent, and rather loose tunic or cyst, and is found in the blood of patients affected with elephantiasis, but only during the night. This disease is manifest in a thick, livid, tuberculate, and insensible condition of the skin, akin to leprosy. It is endemic over the more thickly populated and tropical portions of the globe, and, in its various forms, is very painful, resulting in deformity, and not infrequently in death. The best authorities now believe that various diseases of the lymphatic vessels and glandsas varicose groin glands, lymph scrotum, elephantiasis, and chyluria - are pathologically one and the same, and are due to the presence of this Filaria, which has, in fact, been recorded from South Europe, Asia, Australia, and Brazil; Dr. Araujo having verified at Bahia its occurrence in the mosquito, and otherwise confirmed the observations of Manson and other's in different parts of the world.

Before the young Filariae can undergo their full development, they must first enter the body of the female mosquito (Culex mosquito), which sucks them up in her nocturnal attacks. Within the mosquito they develop in from five to six days, and upon the death of their host, or before, pass into water frequented by the mosquito for purposes of oviposition, and are thus returned, by drinking, to the human stomach, from which they make their way into some lymphatic vessel, where, the sexes meeting, the female remains, perhaps, for years, giving birth to active young.

In the Customs medical report for the halfyear ending March 31, 1882, lately published, and but recently received in this country, Dr. Manson gives the results of some later observations which are full of interest. It seems that the periodicity in the Filaria disease has no connection either with temperature, atmospheric pressure, or light, but must be looked upon as an adaptation of the habits of the parasite to those of the mosquito. 'The conditions for the ingress of the Filariae into the circulation appear to be developed ordinarily during the last few hours of the waking state, and the parasites are eliminated during the last few hours of sleep. Under ordinary conditions of sleeping or waking, the embryos enter the circulation every evening, increase until midnight, and diminish as morning approaches, until they entirely disappear, and are not found from nine A.M. to six P.M. 'This periodicity of the parasite is independent of parturition in the parent, as reproduction is continued during the twenty-four hours.

The importance of thus tracing to their true source diseases whose origin has long been involved in mystery cannot be overestimated; and these facts would seem to give additional reasons for the filtering of drinking-water, and the use of mosquito-bars, in all tropical countries. Dr. Manson suggests that the facts ascertained in this connection may lead to a possible future explanation of the diurnal intermission and remission of fevers of the ague class. 'The most interesting' conclusions that have been forced upon Dr. Manson are, that the presence of the parasite in the human body does not always or necessarily produce disease; and that, when disease is produced, it is by exceptional oviparous reproduction instead of the ordinary viviparous mode.

We give his conclusions in his own words:-

"In the instances in which the parent worm has been discovered, she was found in lymphatic vessels on the distal side of the glands. This has been shown to be in many if not in all cases her normal habitat. Her progeny, therefore, must travel along the afferent vessels, through the glands, and so on to the thoracic duct, and thence into the blood. The long, sinuous, and powerful body of the embryo is well adapted to perform this journey. But suppose, instead of this mature embryo, an ovum is launched into the lymph-stream prematurely, and before the contained embryo has sufficiently extended its chorion, then this passive ovum must certainly be arrested at the first lymphatic gland to which it is carried by the advancing lymph-current. It measures $\frac{1}{\gamma 50}{ }^{\prime \prime} \times \frac{1}{500}{ }^{\prime \prime}$, whereas the outstretched embryo is only about $\frac{1}{3000}$ in diameter. It is much too large to pass the glands; and the embryo, rolled up in its chorional envelope, cannot aid itself. It becomes, in fact, an embolus. Now, Filariae are prodigiously prolific. Myriads of young are expelled in a very short time. I have watched the process of parturition in the minute Filaria corvi torquati. Every few seconds a peristaltic contraction, beginning low down in the uterine horns, and extending to the vagina, expels some twenty or thirty embryos. If this process of parturition occurs prematurely, or peristalsis is too vigorous, and extends to a point high up in the uterine horns, where the embryo has not yet completely stretched its chorional envelope, then ova are expelled. These, as they reach the glands, where the afferent lymphatic breaks up into fine capillary vessels, act as emboli, and plug up the lymph-channels, one after another, until the fluid that carries them can no longer pass. In this way the gland or glands directly connected with the lymphatic in which the aborting female is lodged are thoroughly obstructed. Anastomoses for 
a time will aid the passage of lymph; but the anastomosing vessels will carry the embolic ova as well as the lymph. 'The corresponding glands will then, in their turn, be invaded; and so on, until the entire lymphatic system, connected directly or indirectly with the vessel in which the parent worm is lodged, becomes obstructed.

"This, I believe, is the true pathology of the elephantoid diseases: $1^{\circ}$. Parent Filaria in a distal lymphatic; $2^{\circ}$. Premature expulsion of ova; $3^{\circ}$. Embolism of lymphatic glands by ova; $4^{\circ}$. Stasis of lymph; $5^{\circ}$. Regurgitation of lymph, and partial compensation by anastomoses; $6^{\circ}$. Renewed or continued premature expulsion of ova; further embolism of glands. This process, according to the part of the lymphatic system it occurs in, the frequency of its recurrence, and its completeness, explains every variety of elephantoid diseases." C. V. Riley.

\section{INTERNAL MOLECULAR ENERGY OF ATOMIC VIBRATION.1}

THE object of this paper is to examine at length the relative amount of energy which a molecule may possess with respect to any small degree of freedom of motion which its atoms may have as to each other. The theorem of the virial is applied to this motion of the atoms; and it is found, that in a molecule of a perfect gas consisting of but two atoms, which are at a mean distance, $r$, from each other, and which suffer a small displacement whose mean maximum amplitude is $\delta_{r}$ under the action of elastic forces, the energy of atomic vibration will be to that of translation parallel to any assumed direction in space as $\delta r$ to $r$. It is further shown that this result is of such a character as not to be restricted to molecules of two atoms merely, nor to atoms which are attracted toward their mean position by forces varying simply as the first power of the displacement; so that the result arrived at is of a general nature which may be stated thus : the energy of interatomic vibration depends upon the atomic displacement within the molecule, and in such a way, that, when this displacement is a vanishing quantity compared with the dimensions of the molecule, then this energy of internal vibration is a vanishing quantity compared with the energy of motion of the molecule as a whole.

This result is in confirmation of the results obtained by the author in his previous paper upon 'An extension of the theorem of the virial,' ${ }^{2}$ etc., in which he expressed the opinion that the results there obtained led to the conclusion, that "in case partial constraints not amounting to the loss of entire degrees of freedom are introduced, the energy will no longer be equally distributed among the co-ordinates, but will be influenced by their constraints."

This being in direct contradiction to the conclusions which have been deduced by Boltzmann and by Watson from the discussion of the distribution of energy by the method of generalized co-ordinates, an examination is made of the point in this hitherto accepted theory from which the contradiction arises, and an error is pointed out in the method of employing the fundamental expression for the distribution of velocities. The error is of this nature: the law expressing the most probable distribution of velocities with respect to any single co-ordinate is the same as that of the most probable distribution of errors of obser-

1 Abstract of a paper upon a further extension of the theorem of the virial to the internal molecular energy of atomic vibration. By H. 'T. EDDr, Ph.D., Cincinnati. Read before the Section in physies and chemistry of the Ohio mech. inst. A pril 26, 1883.

2 Sc. proc. Ohio mech. inst., March, 1883; Science, p. 65. vation, and contains a single arbitrary constant, to be determined by the observations themselves. It has been assumed that this constant is the same for each co-ordinate, which is, in effect, assuming the very point to be proved. It is here pointed out, that doing this commits an error of the same nature as is done in assigning equal weights to unlike observations without first showing that their weights are equal.

The computations made by means of the virial show conclusively that the mean energy (i.e., the weight) is not at all the same for one degree of freedom as for another; and, in order to find how one is related to another, it will be necessary to take account of the forces acting, as has been done in this paper and in the previous one.

This extension of the theory leads to numerical results in close accordance with observed values of the specific heats of gases, and their ratio, without previous knowledge of these quantities for any gas; thus computing these quantities for the first time solely from the general equations of mechanics.

\section{ON THE DEVELOPMENT OF CHLORO- PHYLL AND COLOR GRANULES.}

THE view has been generally entertained, based largely on the admirable investigations of Arthur Gris, that chlorophyll-granules are produced by direct differentiation of the protoplasm of assimilating cells. Led by his study of certain protoplasmic bodies in the cells where nutritive matters are stored for future use, and following out a suggestion made by Schmitz in his recent work relative to the assimilating bodies in certain Algae, A. F. W. Schimper (Botan. zeit., Feb. and March, 1883) has made a detailed examination of the origin of chlorophyll-granules, which indicates that the views of Gris are erroneous. At the points of growth examined by him, Schimper uniformly found that well-formed granules already exist, and that, from subsequent division of these, all the chlorophyll-granules are produced. From these, and not, as heretofore believed, from the differentiation of the protoplasmic mass in the cell, arise the granules which later, under the influence of light, take on their characteristic color. One of the most interesting cases reported by him is that of Azolla. The point of growth at the root contains bright green chlorophyll-granules about as large as those in the older parts, and in these granules the process of division is to be distinctly traced.

In those points of growth where the tissues are as yet free from color, he has been also able to follow the division, step by step, up to the production of complete green granules. 'The bodies from which the granules are produced are present, likewise, in all points of growth of seedlings. Just here is found the most interesting feature of this investigation. From these bodies, which he well terms 'plastides,' come three classes of protoplasmic bodies, somewhat resembling one another in shape: namely, 1, the chlorophyll-granules, or chloroplastides; 2, the starch-formers, which, with the allied white or colorless bodies, he calls leucoplastides; and 3 , the bodies which possess colors other than green (for instance, the granules in petals and the like), to which he gives the name chromoplastides. To illustrate this from a single case, we will allude to Impatiens parviflora. The very transparent cells at the point of growth contain plainly visible leucoplastides. In cells of the same age they are of the same size, often constricted, always sharply defined. 'These can be traced by plain transitions into chloroplastides on the young stem and the zone of forming leaves, 